\title{
Two Direct Speeches in the Last Two Poems of the 'Dialogue between a Man and His Ba' (pBerlin 3024, cc. 138-140 and cc. 144-145): A Note of Translation
}

\author{
Stefano Vittori \\ PhD student of Egyptology, Department of Civilization and Forms of Knowledge, the University of Pisa, Italy
}

Copyright $(2018$ by authors, all rights reserved. Authors agree that this article remains permanently open access under the terms of the Creative Commons Attribution License 4.0 International License

\begin{abstract}
Two poetic passages of the 'Dialogue between a Man and his $\mathrm{Ba}$ ' arise problems both in interpretation and in translation: jw mt $m$ hr.j mjn mj kft pt; mj z zht-A1-jm r hmt.n.f (cc. 138-140) and wnn ms nty jm 'h' $m$ wj3 hr rdt d.t stpwt jm r r'w-prw (cc. 143-145): the literal translation of these passages is problematic: "Death is for me today like an unveiling of the sky, like a man + (I acquire? who acquires?) + there + (according to? More than?) He ignores"; "Indeed, who will be there will be standing in (Ra's) boat, allowing that someone gives the offerings there + towards? + the temples". Integration of the verb $d d$, "to say", would transform the former passage into a direct speech and solve the problem: "Death is for me today like an unveiling of the sky, like when a man says: 'I will profit from it' about something he ignores". This integration induces to interpret in the same way also the $h r$ of latter passage: "Indeed, who will be there will be standing on (Ra's) boat, saying: 'There is where one makes offerings be given to me' about temples", shedding better light on the sense of the adverb jm and the preposition $r$. Metric and rhetoric reasons support the integration in the former passage.
\end{abstract}

Keywords Dialogue between a Man and His Ba, pBerlin 3024, Pamherst III, Egyptian Language, Egyptian Philology, Egyptology, Middle Egyptian Literature, Lamentations, Egyptian Poetry

\section{Introduction}

The 'Dialogue between a Man and his Ba' arrived to us thanks to pBerlin 3024 and fragments of pAmherst III. The date of the copy could be the reign of Amenemhet III [1] (1842-1797 a. Chr.).

Two characters, a man and his ba, discuss the sense of the funerary cult from opposite viewpoints. The ba is persuaded that the name of a man lives anyway after its bearer dies (jw grt.k mt.t rn.k 'nh, cc. 36-37), independently of the religious comforts, which does neither bear any benefit to him who offers them nor to him who receives them. The fate of the altars of rich people is to be abandoned, which is exactly the same fate of the corpses of that poor people who have been "buried" by the inundation, without funeral ( 'b3w jry wš. $w$ mj nnjw mtw hr mryt $n g 3 w$ hry-t3, cc. 63-67), concluding his statement suggesting to dedicate himself to enjoy life and get rid of his continuous anxieties ( $\check{m} m s$ hrw nfr smh $m h$, c. 68). But the man is not persuaded: according to him, a death not endowed with funeral would cause his name to be "washed away", like the water of the inundation washes away the dirt of the ground in the heat of summer ( $m . k b h r n . j, \mathrm{cc} .86-103)$. On the other hand, enjoying life makes no sense to him, for life is now to him nothing but loneliness ( $\underline{d} d . j n m m j n$, cc. $103-130)$, the only thing he can desire by now is death (jw $m t m$ hr.j $m j n$, cc. 130-142), because a righteous death can give to a man the chance to be with gods and live forever thanks to funerary cult (wnn ms nty jm, cc. 142-147). The ba does not reply to these statements; he just limits himself to assure his counterpart that anyway he will be with him in the hereafter (s3h h'w.kt3 (...) jr.n dmj n zp, cc. 152-154). Because of intertextual elements, style, characters, the topic, the work seems strictly related with the Dialogue between Khakheperraseneb with his Heart: it has been supposed that this dialogue, of which only the last columns survive, is the prosecution itself of the Dialogue between Khakheperraseneb and his hearth, of which, on the contrary, we have only the initial part [2].

In this occasion, we will deal with two passages of the penultimate and of the last poem of the man's speech, where he says that death is for him the most desirable thing in the world (cc. 130-142) and where he expresses his good feelings about the hereafter (cc. 142-147). 


\section{The First Passage}

The third poem consists of five nine-stressed couplets of Mathieu's metric system [3] followed by a quatrain whose first verse is the refrain and the other three are a nine-stressed triplet of the same type:

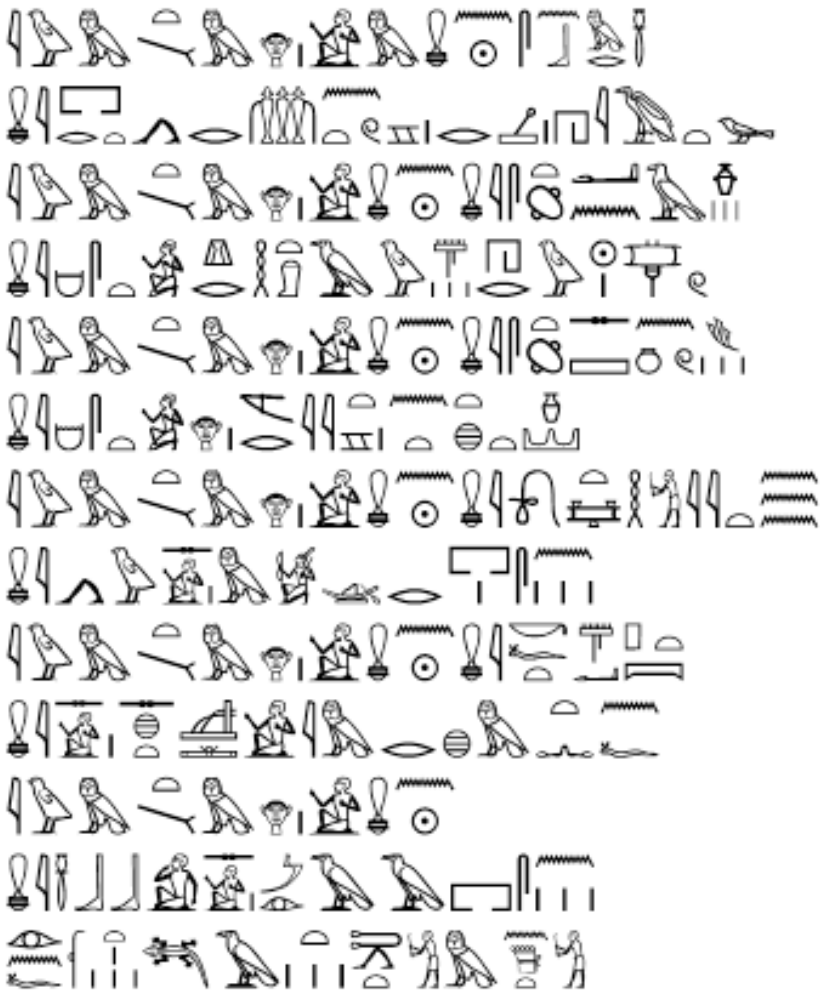

Figure 1. Hieroglyphic transcription of Hieratic of pBerlin 3024, cc. 130-142. Original in columns leftwards

${ }^{130} j w m t|m h \underline{r} . j| m m j n|| s n b \mid{ }^{131} m r$

mj prt |r hntw ||rs3| ${ }^{131-132}$ hjmwt

Death is for me today health for who is ill,

like going out in the courtyard after an illness ${ }^{(?)}$.

jw $m t|m h \operatorname{hr} . j| m j n|| m j$ st ${ }^{132-133}$ ntyw

mj hmst $|\underline{h} r \underline{h t} 3 w|\left|{ }^{134} h r w\right| \underline{t} 3 w$

Death is for me today like scent of myrrh grains, like sitting under a tent in a windy day.

$j w m t\left|m h r_{. j}\right| m j n||{ }^{135} m j s t \mid z \check{s} n w w$ mj hmst | $\underline{h r} m r y t||{ }^{136} n t \mid t h t$

Death is for me today like scent of lotus flowers, like sitting on the shore of the (Land of) Drunkenness.

$j w m t|m h r . j| m j n|| m j{ }^{137} w 3 t \mid h y t$

$m j j w|z| m m \check{s}^{\prime} \|{ }^{138}$ r pr.sn

Death is for me today like the way of the stream, like when a man comes back home from a navigation.

$j w m t|m h r . j| m j n|| m j{ }^{139} k f t p t$

$m j z|z h t ? j ?|{ }^{140} j m|| r$ hmt.n.f

Death is for me today like an improvement of the sky, like a man ... there ... that which he ignores. jw $m t|m h \underline{h} . j| m j n||$

${ }^{141} m j 3 b b|z||m 33|$ pr.sn

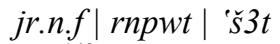

$j t \mid{ }^{142} m n \underline{d r} t$

Death is for me today

like when a man wishes to see his home

after he spent many years

held in a prison.

The terms of comparison with death are all simple enough: wealth, paradise, way home etc. Nonetheless, one of these comparisons appears to be less obvious and arises controversies in translation. It is that of cc. 139-140:

$$
\text { mi z zht ?j? jm } r \text { hgmt.n.f }
$$

The verb sht (here $z h t$ because of sandhi) means "to catch, trap". Weill [4] translates the verb as a participle the verb of which is understood as "to be instructed" from the co-text, while he considers $j m$ as an argumentative. Faulkner [5] refuses to translate the sentence due the doubtful meaning of the verb, but he is sure too that the verb is a participle. Barta [6] translates it as a participle, jm as an instrumental $m$ and reads $r$ as $r$ '. So, the meaning becomes abstract if compared with the sensorial concreteness of the other comparisons. Lohmann [7] reads the succession $j-m-r$ as jmy-r' and understands it as the object of a $z h t$, participle too, which nevertheless remains abstract: in her interpretation, hmt.n.f is a perfective passive participle with agent, with feminine ending since the noun should be jmyt-r', "what is in the mouth" (WB.I.74.12). Mathieu [8] interprets the verb as "to understand" too, but the participle is considered as a noun and translated a nomen actionis. In both Barta's and Lohmann's interpretations an ideographic mark Z1 is to be integrated beside $r$ and in all these interpretations the human mark A1, determinative of person or ideogram of.$j$, is to be expunged.

The scribe of the Dialogue is not exempt from omissions: in c. 15 , he writes $t k$. $f$ for $t k n$. $f$, in c. $693 p$. $f$ for $3 t p$. $f$, in c. 87 $3 s . w$ for $3 p s w$; nevertheless, among the nouns which use $\mathrm{Z} 1$ repeated more than once in the text, $d m j$, "town" (cc. 38, 102, 154), hr , "face" (cc. 39?, 79, 118, 130, 132, 134, 136, 138, 140), hrw, "day" (cc. 15, 68, 88, 90), jb, "heart" (cc. $38,52,85,105,118,120,121,124,126,128)$, ntr , "god" (cc. 24, 30, 63, 142), $\underline{h t}$, "belly" (cc. 9, 30), pr, "house" (cc. $83,138,141,145), r$ ', "mouth" (cc. 4, 86, 145), r' "Ra, sun" (cc. 25, 60), z, "man" (cc. 31, 58, 105, 110, 119, 121, 137, 139, 141), t3, "earth" (cc.34, 42, 64, 78, 109, 112, 122, 129, 152 ), the only ones which lack that stroke are $p r$, "house", in two of four attestations (cc. 83, 138) and $n \underline{t} r$ in one of four (c. 142, but there it is a compound noun: ntr ' $n h$, "living god"); while the noun $r$ ', which in the first two occurrences appears in the frame formula $j w w p . n \mathrm{X} r$ ' $\mathrm{X} n$

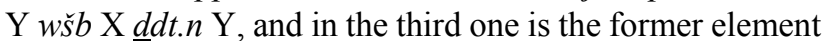
of the plural of the compound noun $r$ '-pr, "temple", never lacks the vertical mark on its left (cfr. attestations of these lemmata on www.papersesh.com $\rightarrow$ search $\rightarrow$ thesaurus $\rightarrow$ 
by source $\rightarrow$ pBerlin 3024$)$. Hence, a $r$ "mouth" with fallen $\mathrm{Z} 1$ stroke in c. 140 would be unparalleled in the text.

The scribe is very precise about the personal pronoun of first person: only twice in the whole text he omits it, c. 12, n $y t(. j) n . f$, "(he [= the ba] pulls me to death) while I did not yet reach it", and c. 13, $h r h 3^{\circ}(. j) h r h t$, "(he [= the ba]) is setting me on fire". In no case he writes it where it is not needed; and the same can be said about the same grapheme used as a determinative. For these reasons, I think that he is trustworthy when he adds it to the word of c. 139. Hence, the sentence is:

$$
\text { mj z zht.j jm } r \text { h̆mt.n.f }
$$

Naturally, the central lemma is sht. The meaning "to understand", proposed by Barta and Lohmann, can be induced by the abstract determinative of the rolled papyrus $<$ Y1 >; nevertheless: 1) it has not only an abstract meaning, but it is also a positive mark; 2) it does not seem to me that the meaning "to understand" can be attested elsewhere for this verb: it is rather used stricto sensu as a verb related to haunting, cp. Pyr. 62c: $m 3 . k n$ prt jm.k: h(wj).s sht jm, "Look at what came forth from you: it shall hit the one who is netted in it (Seth)" [9], or lato sensu as an action against somebody; cfr. Ptahhotep pPrisse VI.7 [10]: sht,$j$ r.j $\underline{d s . j}$, "I trap myself", possibly extensible to the meaning "to earn", cfr. the Learning of the Papyrus of the Ramesseum II Vso II.4 [11]: $r h-h t h r(d d)$ sht.j $m j$ hm, "the wise man says: 'I earn as an ignorant man"”; 3 ) in the rest of the poem, death is compared with extremely concrete objects and actions, which bear an immediate sensorial pleasure. This concreteness holds true both for the preceding verses and for those which follow this one: it is not very clear why, only and exactly in this passage, the Author would have passed to an abstraction. This is a very stylistically coherent poet from a semantic viewpoint. For example, when he dreads the oblivion of his name, he chooses a key verb for the whole passage, i.e. a verb describing the inundation while washing the surfaces of the land in summer time. This verb suggests a series of concrete themes: odor of birds, fishermen and crocodiles as a counterbalance of that of myrrh and lotus in the passage under discussion. Nonetheless, in the sixth couplet, the noun st, "odor", is substituted by an isometric one, $z t$, "woman", which with hmt is a compound noun the latter element of which replaces the genitive of the preceding verses. From this point on, the poet passes from a concrete to an abstract level: his name will be washed away not anymore from a surface, but from the collective memory one would say 'removed' -, and not anymore for aesthetic reasons (a bad smell) in instinctively abominable subjects (birds, fishermen, crocodiles), but for ethical reasons (conjugal infidelity and its fruit; politic betrayal) in intellectually abominable subjects (an adulterer woman, an illegitimate child, a city which betrayed its own sovereign). But once he passed from concrete to abstract, he remains in this letter and in this latter he concludes. Since on the contrary in this poem he remains in a concrete level till the end, it can be supposed that he remains in it in that single passage too. Hence, we should give a concrete meaning to that $s h t$ and, with the rolled papyrus, a positive nuance.

In PRam. II, Vso II.3, one reads: $m . t n h m w n h m n r h$ tnw.f: "Lo, as for the goods of the ignorant man, their number is unknown!"; for Vso II.4, $r h \underline{h} h t h r(\underline{d} d) s h t . j m j$ $h m$, in a first hypothesis of translation, the direct speech could end with sht.j: "the wise man says: 'I will trap' as the ignorant man (says it)". Nonetheless, if one compares this passage with the preceding one, and if one considers how the equality in condition between wise and ignorant man is cause of complaint in Ipu-ur, PLeiden 344 Rto IV.7-8 [12], dd.tw w3 $r$ st-št $3 w$ m.k $s w$ m-' hm $s w$ mj $r h$ sw, "One conspires against the place of secrets: lo, it (that place) is in the hand of him who ignores it like in that of him who knows it", it seems more likely to me that the direct speech is extended to the following clause: sht.j mj hm: "(Alas!) I earn as much as the ignorant man!". The verb sht must necessarily refer to $h m w$, which indicates "remuneration", an economic benefit (WB.II.490.5), the infinity of which for the ignorant man is object of complaint in PRam. II Vso II.3: "to obtain economic benefits" is hence to be understood as the meaning of "to catch" in this case. The wise man of the PRam. II says: "Alas! My benefit is as big as that of the ignorant man! So what is my wisdom aimed to, since it gives me no advantage on those who are not provided with it?". In the cited passage of Ptahhotep, a criminal is sure to conquer a high position thanks to his dishonesty, but since crime does not pay ( $\mathrm{p} p 3 w \underline{d} 3 y$ t mjnj $z p . s$, VI.6), he ends up by saying: (jw.j) sht.j r.j $\underline{d} s . j$, "I trap myself!". Both Ptahhotep's passage and that of pRam. II tell that the verb can occur in direct speech in exemplar situations of the subject depending on his conduct.

In this poem, the latter hemistich of the former verse of each couplet introduces a comparison which is developed or varied in the latter verse. In the first one, the poet begins with wealth for an ill person and amplifies this topic with the idea of a person who can at last go out in his courtyard after an illness which, as one can easily imagine, forced him to stay closed in his bedroom for a while. In the second one and in the third one, he speaks about scent, the former linked to a sense of serenity and protection, the latter to paradise. In the fourth one, he speaks about "sailing downstream" (w3t hyt, "the way of the stream"); then he describes a man who comes back home after an expedition by sea or by river. The two verses together describe a placid way back after a trip imagined as stormy and dangerous. What follows is the couplet under discussion. Finally, the poet concludes with the quatrain introducing the image of a prisoner on his way home, with a variation of the image of the fourth couplet. As a matter of a fact, it seems that there is always a link between the former and the latter verse of each couplet: what is said in the latter is always an explanation or a variation of what has been said in the former. Hence, here too, there must be some 
relationship between $k f t p t$, a sudden improvement of the sky after the rain, and what follows. If one considers the verb sht in the already seen nuance "to earn, obtain benefits from something", one can imagine that the benefit of the man subject of the sentence is a pleasing surprise.

The presence of $z$ indicates a third person, different from the speaker, who compares himself with that third person, while the first person, sht.j, indicates a direct speech: one should individuate its right border in order to understand whether $r$ is a preposition introducing a complement of a verb or not. Since the agent of the participle is a third person, the direct speech must end immediately before the preposition introducing that participle: hence, the direct speech is only sht.j jm.

In the immediate co-text of the poem, the comparison is developed by means of $m j$ followed by a suffixed verb with a nominal subject $z$ : in this verse, one reads $z$ too, but the verb is lacking. If it is restituted, the poem ends with a group of three perfectly homogeneous couplets (the last as the initial part of a final quatrain) from the viewpoint of both metric and rhetorical structure:

$$
\begin{aligned}
& \text { jw } m t \mid m \text { hr.j| mjn }|| m j w 3 t \text { hyt } \\
& \boldsymbol{m j} \mathbf{j w}\left|\boldsymbol{z}^{\prime}\right| m \mathbf{m s}^{\prime} \mid \text { r pr.sn } \\
& \text { jw } m t\left|m h r_{. j}\right| m j n \| m j k f t p t \\
& \text { mj [미] } \mid \boldsymbol{z}^{\prime} \text { | 'zht.j im' | r hmt.n.f } \\
& \text { jw } m t \mid m \text { hr.j|mjn } \| \\
& \boldsymbol{m j} \mathbf{3} \boldsymbol{b} \boldsymbol{b}\left|\boldsymbol{z}^{\prime}\right||m 33| \text { pr.sn }
\end{aligned}
$$

Death is today for me like a downstream trip,

like when a man comes back home after a navigation.

Death is today for me like an improvement of the sky,

like when a man says: 'I will earn from it!' of what he does not yet know.

Death is today for me like when a man wishes to see his home.

The meaning of the couplet under discussion is clearly positive: the poet is not speaking about an inconsiderate person, who foresees benefits in events whose possible evolutions are unpredictable to him. The verb $\underline{d} d$ in aoristic and gnomic expressions, both in its complete form $h r \underline{d} d d$ and in the shortened $h r$, indicates the right, the duty, and the necessity of saying. In this meaning, one finds it both in Ptahhotep's iw.f $\underline{d} d . f$ and in pRam. II $r \underline{h}-\underline{h t} h r(\underline{d} d)$, where this verb indicates a matter of a fact caused by an incontrovertible reality: from a literal viewpoint, one must translate it as "he says"; but from a semantic one, it should rather be understood as "he ends up by saying", "he can, must say", "there is nothing left for him but saying" and the like.

\section{The second Passage}

The following poem (cc. 142-147), three nine-stressed couplets, concludes the man's last speech, expressing his positive feelings about the hereafter:

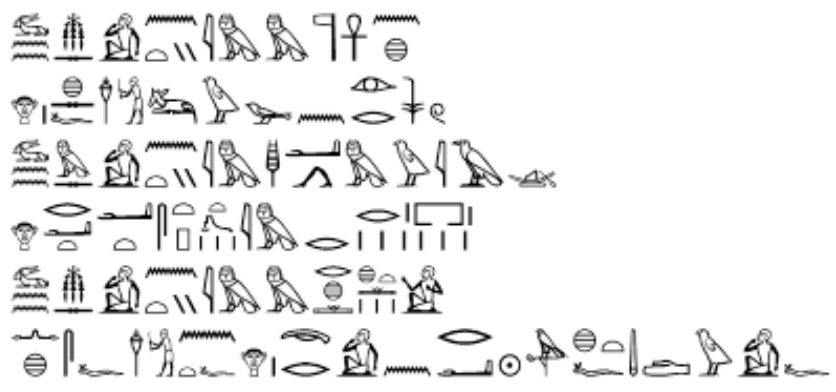

Figure 2. Hieroglyphic transcription of Hieratic of pBerlin 3024, cc. 142-146. Original in columns leftwards.

$$
\begin{aligned}
& { }^{142} w n n|m z| \text { nty jm }|| m \text { ntr } \mid \text { 'nh } \\
& { }^{143} h r h z f|j w \| n j r| s w
\end{aligned}
$$

Indeed, who will be there will be a living god, who will repel evil to him who did it.

$$
\begin{aligned}
& \text { wnn }|m z|{ }^{144} \text { nty jm }|| h^{\prime} \mid m \text { wj3 } \\
& \text { hr rdt } \mid \text { d.t }|| \text { stpwt }{ }^{145} j m \mid r r^{\prime} w \text {-prw }
\end{aligned}
$$

Indeed, who will be there will be standing on the boat, making the offerings to be given towards the temples.

$$
\begin{aligned}
& \text { wnn }|m z| \text { nty jm } \| m r h \mid{ }^{146} h t \\
& n \text { hsf.n.t.f } \mid \text { hr spr } \| n{ }^{147} R^{\prime} \mid \text { hft } m d w . f
\end{aligned}
$$

Indeed, who will be there will be a savior

who will not be driven away while referring to Ra every time he speaks.

It does not seem unlikely that what has been proposed for the syntactically doubtful passage discussed above can be proposed for the second couplet of this other poem too, where the sequence $<\mathrm{D} 36: \mathrm{X} 1>$ has the reading $\operatorname{d.t}(w)$, "that one gives (= it is given)", so that the meaning is "making the offerings be given". The sentence is only apparently (grammatically) sensed: why is the dead the one who manages the delivery of offerings? This is a duty of the funerary priest, the $h m-k 3$. He should be rather the one whom they are given to. Furthermore, even supposing that, until stpwt, this interpretation is correct, which is the sense of $\boldsymbol{r} r$ 'w-prw? Offerings should be put in the temples, given to the temples, but not put or given towards, for, on the temples. Suys [13] does not comment his translation of this passage, but he seems to consider $r$ as a synonym of $n$, and so do Weill [4], Lohmann [7] and Mathieu [8]. Faulkner [5] translates more literally "to be given (...) for", but which is the meaning of such a verb like "to give for"?

The preposition $r$ can mean also "on", but if it meant "on", then here there should be rather " $b 3 w$, "altars", than "temples", and even in this case one would expect $h r$ rather than $r$. Both the presence of $j m$ and that of $h r$ induce to think that this is the same situation seen above: the person who will be in the hereafter will have the right to speak of $(r)$ the temples $\left(r^{\prime} w\right.$-prw) as the place where $(j m)$ offerings are given (to him).

This interpretation is supported by metrical reasons too. The preposition $h r$, when hiding $\underline{d} d$, is proclitic to the following word as if it were a normal preposition and not a 
verbal progressive mark, not so differently from Coptic çe, "that" $=(r-) \underline{d} d$. The whole couplet is hence to be read: wnn $|m z|^{144}$ nty jm ||$h^{\circ} \mid m$ wj3

$h r(\underline{d} d)$ 'Rdt $\mid$ d.t $t(w)\left|s t p w t^{145} j m^{\prime}\right| r r$ 'w-prw

Indeed, who will be there will be standing on the boat,

saying of the temples: 'There is where offerings are delivered (lit. "made be given") (to me)'.

\section{Conclusions}

Death is a mystery for everyone, but somebody feels that, whatever hides behind it, it will be good for him: not just because his life is so terrible that every alternative is desirable, but mainly because, although nobody knows with certainty what will happen after death, everyone who is a principled person has the right to feel that it will be nothing bad.

The dead who will receive the traditional funerary cult will be able to say that a part of the offerings will be destined to him. The righteous person, when dying, has not only the feeling, but also the certainty that his memory not only will not be lost, but that the official cult will make him immortal.

The adverb $j m$ and the preposition $r$ in these two passages are both problematic if interpreted as having other functions than as respectively a local adverb and a marker of the complement of argument. The first $j m$ is death; the second $j m$ is the temple: together, they are the two semantic poles of the entire work. The two prepositions $r$ explain what these two jm are: the unknown in one case, the official cult in the other. This is how these two subjects are treated throughout the work.

The value given to these two words makes it necessary to see two direct speeches. They are what the protagonist hopes to say someday; but by now he is forced to say something completely different, in the excruciating description of a life and of a society where there is nothing good to expect for the righteous man. This emotional situation arises in the man a fundamental opposition: his ba and himself. This implies other dissociations: society and individual, materialism and religion, reason and emotion, all-day life problems and ideology. All these polarities are highlighted by the style itself: the man is a poet; the ba is a philosopher. The two $\mathrm{jm}$ 's are the final dissociation: death, as a physical phenomenon, and temple, as the place where the feelings arisen by this phenomenon are sublimated. In this sublimation, the protagonist hopes to have found a way to solve all his inner lacerations. The ba is not persuaded at all of this solution: according to him, these problems will be solved, and both of them will be reconciled to one another, only after death and only because of death itself, with no need for sublimations of any sort.

\section{REFERENCES}

[1] J. P. Allen, the Debate between a Man and his Soul: a Masterpiece of Ancient Egyptian Literature, Leiden, Boston, 2011.

[2] C. Barbotin, Le dialogue de Khâkheperrêseneb avec son Ba: tablette British Museum EA 5645/Ostracon Caire JE 50249 + Papyri Amherst III \& Berlin 3024, Revue d'Egyptologie, No. 63, 2012, 1-20.

[3] B. Mathieu, Etudes de métrique égyptienne I-IV, Révue d'Egyptologie, No. 39, 1988, 63-82; No. 41, 1990, 127-141; No. 45, 1994, 139-54; No. 48, 1997, 109-152.

[4] R. Weill, Le livre du 'Désespéré': le sens, l'intention et la composition littéraire de l'ouvrage, Bulletin de l'Institut Français d'Archéologie Orientale, No. 45, 1947, 89-154, 129.

[5] R. O. Faulkner, The man who was tired of life, Journal of Egyptian Archaeology, No. 42, 1956, 21-40, 29, 30.

[6] W. Barta, Das Gespräch eines Mannes mit seinem BA (Papyrus Berlin 3024), Berlin, Hessling 1969.

[7] K. Lohmann, Das Gespräch eines Mannes mit seinem Ba, Studien zur Altägyptischen Kultur, No. 25, 1998, 207-236, 224, 225.

[8] B. Mathieu, Le dialogue d'un homme avec son âme: un débat d'idées dans l'Egypt ancienne, Egypt, Afrique \& Orient, No. 19, 2000, 17-36, 24.

[9] J. P. Allen, the Ancient Egyptian Pyramid Text, 23, and 62, n. 20.

[10] G. Jéquier, Le papyrus Prisse et ses variantes, Paris, Geuthner, 1911, Pl. III.

[11] A. Gardiner, the Ramesseum Papyri. Plates, Oxford, 1955, 8-9 and P1. III-VI.

[12] A. Gardiner, The admonitions of an Egyptian sage, Hildesheim, Georg Olms, 1969, 37-38.

[13] E. Suys, Le dialogue du désespéré avec son âme, Orientalia, No. 1, 1932, 57-74, 70.

[14] A. Erman, the Literature of the Ancient Egyptians: Poems, Narratives, and Manuals of Instruction, from the Third and Second Millennia BC, London 1927, 86-92.

[15] R. Williams, Reflections on the Lebensmüde, Journal of Eastern Studies, No. 48, 1962, 49-56. 